\title{
Bilateral Chorea Associated with Acute Caudate Nucleus Infarctions
}

\author{
Yang Qu*(D), Hang Jin*, Zhen-Ni Guo, Fu-Liang Zhang (iD, Jia Liu, Hai-Qiang Qin, \\ Yi Yang
}

Keywords: Caudate nucleus infarctions, Chorea

doi:10.1017/cjn.2019.50

Can J Neurol Sci. 2019; 46: 451-452

A 60-year-old woman was admitted to our emergency room with acute-onset chorea of all four extremities (see video in supplementary material). Her medical history was unremarkable apart from hypertension. A neurological examination revealed no abnormalities apart from involuntary movements. Brain magnetic resonance imaging showed acute bilateral infarctions in the head of the caudate nucleus (Figure 1A and B). Magnetic resonance angiography revealed that both anterior cerebral arteries (ACAs) originated from the right internal carotid artery (Figure $1 \mathrm{C}$ and D). We started administering oral antiplatelets (aspirin at $100 \mathrm{mg}$ / $\mathrm{d}$ and clopidogrel at $75 \mathrm{mg} / \mathrm{d}$ ) and oral atorvastatin $(40 \mathrm{mg} / \mathrm{d})$ for 21 days after the final diagnosis, and the patient's chorea signs spontaneously improved. The aspirin and atorvastatin continued to be administered after discharge. At the 6-month follow-up assessment, the patient reported no residual symptoms.

Bilateral caudate nucleus infarctions are rare; a retrospective analysis of 240 cases revealed that the average incidence of bilateral caudate nucleus lesions was $1.6 \%{ }^{1}$ The formation of this infarction type is mainly related to the vascular anatomy of the caudate head and variations of anterior circulation. Previous studies reported that the caudate head receives its blood flow from three principal sources ${ }^{2,3}$ : (1) the recurrent artery of Heubner, a directly penetrating artery that originates from the proximal A2 segment, A1-A2 junction, or distal A1 segment of the ACA; (2) the anterior lenticulostriate arteries, which originate from the A1 segment of the ACA; and (3) the lateral lenticulostriate arteries, which originate from the middle cerebral artery. Because our patient's left ACA lacked an A1 segment, the right ACA's A1 segment bilaterally supplied the recurrent arteries of Heubner and the anterior lenticulostriate arteries. Consequently, occlusion of these arteries is regarded as a cause of bilateral caudate head infarctions. $^{2}$

Post-stroke movement disorders are diverse. ${ }^{4-6}$ A previous study ${ }^{6}$ showed that chorea is the second most common postischemic stroke (IS) movement disorder, accounting for approximately $17.4 \%$ of all post-IS movement disorders. However, nearly $90 \%$ of post-IS chorea cases involve hemichorea, which makes post-IS bilateral chorea a rare occurrence. ${ }^{5}$ Previous studies of patients with bilateral caudate head infarctions have reported diverse potential neurobehavioral symptoms such as confusion, ${ }^{7}$ dementia, ${ }^{7}$ apathy, ${ }^{3,7}$ delirium, ${ }^{8}$ disorientation, ${ }^{9}$ confabulations, ${ }^{9}$ mutism, ${ }^{3}$ depression, ${ }^{3}$ decreased recent memory, ${ }^{10}$ and reduced spontaneity. ${ }^{10}$ The most common behavioral disturbance appears to be abulia. ${ }^{3}$ However, in the present case, the patient did not experience any of the aforementioned symptoms or neurobehavioral disturbances other than choreic movements of the four extremities, as vividly demonstrated in the video in supplementary material.

Hemichorea has been classically associated with vascular lesions affecting the contralateral or ipsilateral caudate nucleus, lentiform nucleus, or thalamus. ${ }^{1,4,5}$ The bilateral motor corticostriato-pallido-thalamo-cortical loop and related neural pathways might have been involved in the chorea of all four limbs observed in our patient. ${ }^{4}$

\section{Supplementary Material}

To view supplementary material for this article, please visit http://dx.doi.org/ 10.1017/cjn.2019.50.

\section{ACKNOWLedgements}

This research was supported by the National Key R\&D Program of China (2016YFC1301600), JLUSTIRT (2017TD12) to Yi Yang.

\section{Disclosures}

The authors declare no conflict of interest.

\section{Statement of Authorship}

Drafted the manuscript: YQ, HJ, and FLZ. Acquisition of data: YQ. Analysis or interpretation of data: HJ, ZNG, and FLZ. Image and video analysis: JL and HQQ. Study concept and design: YY.

From the Stroke Center, Department of Neurology, the First Hospital of Jilin University, Chang Chun, Jilin, China (YQ, HJ, FLZ, YY); Clinical Trial and Research Center for Stroke, Department of Neurology, the First Hospital of Jilin University, Chang Chun, Jilin, China (ZNG, YY); Shenzhen Institutes of Advanced Technology, Chinese Academy of Sciences, Shenzhen, China (JL); Department of Neurology, Beijing Tiantan Hospital, Capital Medical University, Beijing, China (HQQ)

Received January 4, 2019. Final Revisions Submitted April 1, 2019. Date of ACCEPTANCe ApriL 6, 2019.

*These authors contributed equally to the manuscript.

Correspondence to: Yi Yang, Stroke Center, Neuroscience Center \& Clinical Trial and Research Center for Stroke, Department of Neurology, the First Hospital of Jilin University, Xinmin Street 71\#, 130021, Changchun, Jilin, China. Emails: doctoryangyi@163.com; doctor_yangyi@hotmail.com 


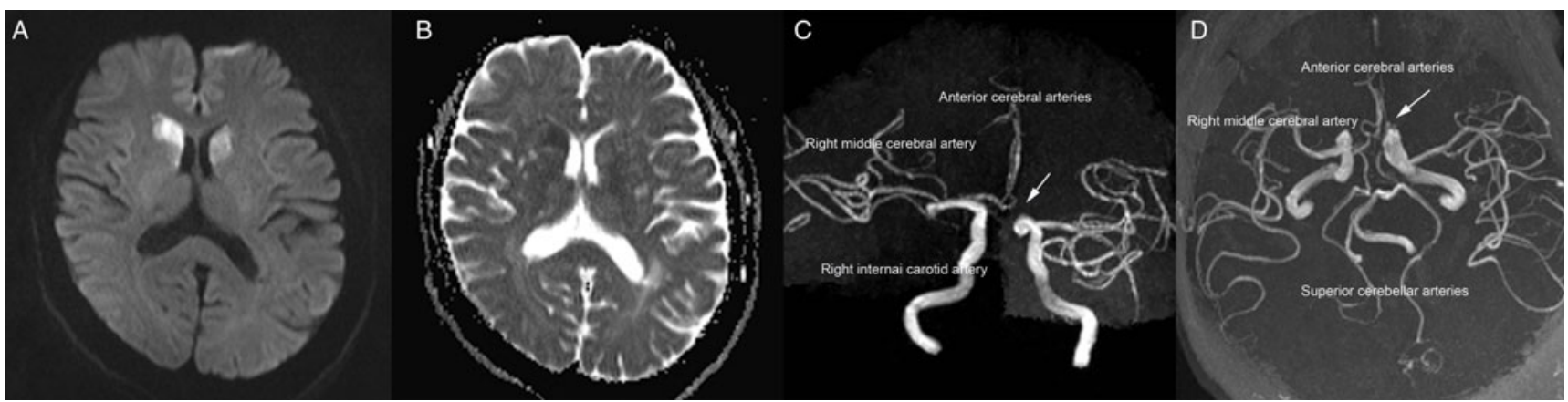

Figure 1: (A) Diffusion-weighted imaging conducted 2 days after symptoms onset revealed a high-intensity signal in the bilateral head of the caudate nucleus. (B) The apparent diffusion coefficient values within the bilateral head of the caudate nucleus were low. (C and D) Magnetic resonance angiography revealed variations in the anterior cerebral arteries (arrows).

\section{REFERENCES}

1. Bhatia KP, Marsden CD. The behavioural and motor consequences of focal lesions of the basal ganglia in man. Brain. 1994;117 (Pt 4):859-76.

2. Kumral E, Evyapan D, Balkir K. Acute caudate vascular lesions. Stroke. 1999;30:100-8.

3. Fukuoka T, Osawa A, Ohe Y, Deguchi I, Maeshima S, Tanahashi N. Bilateral caudate nucleus infarction associated with a missing A1 segment. J Stroke Cerebrovasc Dis. 2012;21:908 e911-2.

4. Mehanna R, Jankovic J. Movement disorders in cerebrovascular disease. Lancet Neurol. 2013;12:597-608.

5. Caproni S, Colosimo C. Movement disorders and cerebrovascular diseases: from pathophysiology to treatment. Expert Rev Neurother. 2017;17:509-19.
6. Suri R, Rodriguez-Porcel F, Donohue K, et al. Post-stroke movement disorders: the clinical, neuroanatomic, and demographic portrait of 284 published cases. J Stroke Cerebrovasc Dis. 2018;27:2388-97.

7. Mendez MF, Adams NL, Lewandowski KS. Neurobehavioral changes associated with caudate lesions. Neurology. 1989;39:349-54.

8. Narumoto J, Matsushima N, Oka S, et al. Neurobehavioral changes associated with bilateral caudate nucleus infarctions. Psychiatry Clin Neurosci. 2005;59:109-10.

9. den Heijer T, Ruitenberg A, Bakker J, Hertzberger L, Kerkhoff H. Neurological picture. Bilateral caudate nucleus infarction associated with variant in circle of Willis. J Neurol Neurosurg Psychiatry. 2007;78:1175.

10. Mrabet A, Mrad-Ben Hammouda I, Abroug Z, Smiri W, Haddad A. Bilateral infarction of the caudate nuclei. Rev Neurol (Paris). 1994;150:67-9. 